\title{
Parasitologische Untersuchungen an alpinen Kleinsäugern: Parasitische Milben (Acari)
}

\author{
von \\ Volker MAHNERT ${ }^{1}$
}

Mit 2 Abbildungen

EINLEITUNG

Im Rahmen einer Dissertation über Ento- und Ektoparasiten alpiner Kleinsäuger (MAHNERT 1970) wurden zwischen Herbst 1966 und Sommer 1968 in drei Untersuchungsgebieten Nordtirols (Umgebung von Innsbruck, 600$1400 \mathrm{~m}$; Umgebung von Obergurgl, Ötztaler Alpen, 1800-2750 m; Kühtai, Stubaier Alpen, 2000-2400 m) von 664 Kleinsäuger (23 Arten) auch parasitische Milben abgesammelt. Von diesen Wirten wurden 2243 Acari der Parasitiformes, Sarcoptiformes und der Trombidiformes excl. Trombiculidae nachgewiesen. Die Trombiculidae konnten nicht regelmässig gesammelt werden, einzelne Ergebnisse werden hier angeführt; die Ixodidae werden anderenorts veröffentlicht (MAHNERT, in Druck). Für die hilfreiche Bestimmung des hier besprochenen Materials sowie für Literatur und Hinweise danke ich Herrn Doz. RNDr. M. MrCiAK, Bratislava, aufrichtig; ebenso gilt mein Dank Herrn Prof. Dr. O. KePKa (Graz), der die Determination der Trombiculidae übernommen hatte.

\footnotetext{
${ }^{1}$ Aus dem Institut für Zoologie und der Alpinen Forschungsstelle der Universität Innsbruck.
} 
TABELLE 1

Die häufig gefangenen Kleinsäuger-Arten und ihre Befallsdichte.

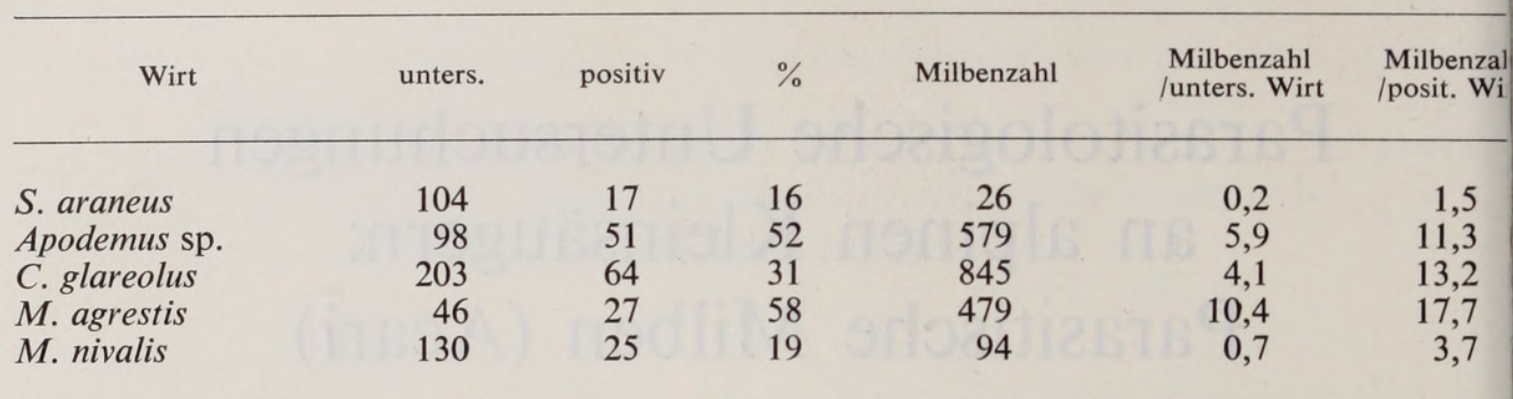

Die zwei (drei ?) Arten der Gattung Apodemus werden gemeinsam behandelt da die systematische Trennung der Arten noch nicht eindeutig vorgenomme werden konnte. Neben den in Tabelle 1 angeführten Arten konnten noch folgend 7 Kleinsäuger- Arten als Wirte festgestellt werden (die erste Zahl in der Klamme gibt die Zahl der positiven Wirte, die zweite Zahl die der untersuchten Wirte an) Myotis oxygnathus (2/6), Nyctalus noctula (3/6), Talpa europaea (1/4), Neomy, anomalus (2/2), Sorex alpinus (4/11), Pitymys subterraneus (6/12), Microtu arvalis (2/2); keine Milben konnten von Crocidura suaveolens mimula (5), Sore minutus (11), Mus musculus (4) und Rattus norvegicus (9) abgesammelt werden ebenso erwiesen sich die Gliridae (13) sowie Myotis myotis (2), Rhinolophu. ferrumequinum (1) und Rh. hipposideros (1) als negativ.

Aus der vorstehenden Tabelle 1 ist ersichtlich, dass Microtus agrestis sowoh in der durchschnittlichen Befallsintensität wie auch in der Befallsextensität di höchsten Werte erreicht. Von 104 Sorex araneus konnten dagegen nur 26 Acar abgesammelt werden, nur $16 \%$ erwiesen sich befallen. KEPKA (1964) vertritt dis Meinung, dass Soriciden allgemein durch Geruchtsstoffe oder andere chemisch Reize für Trombiculiden wenig attraktiv sind, vielleicht spielt ein solcher Faktol auch hier eine Rolle. Allerdings wur de in zahlreichen vorliegenden Untersuchunger ein wesentlich höherer Befallsprozentsatz der Waldpitzmaus festgestellt (z.B EDLER 1969, MrCIAK et al. 1966), sodass die von mir erreichten Ergebnisse viel. leicht auf andere Faktoren (rasche Abkühlung der Wirte z.B.) zurückzuführer sind.

Es war versucht worden, ein ungefähr gleichwertiges Material aus den Tallagen und aus der subalpinen und alpinen Stufe zu sammeln. So konnten von

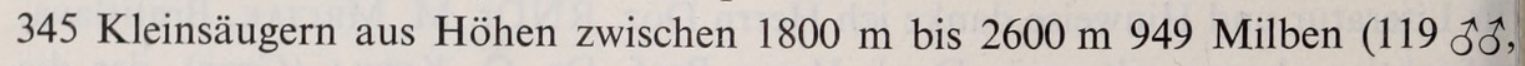
720 우, 107 NII 3 NI), von 319 Wirten aus Tallagen (bis $1000 \mathrm{~m}$ ) 1251 Milben (108 ડิరో, 976 우, 157 NII 9 NI) abgesammelt werden. Im Material der subalpinen und alpinen Stufe dominierte eine Art, Laelaps clethrionomydis, die $60,2 \%$ aller gefundenen Exemplare darstellt, gefolgt von Hirstionyssus tatricus $(12,6 \%)$, 
Iaemogamasus nidi $(7,4 \%)$, Laelaps hilaris $(7,2 \%)$ und Hyperlaelaps arvalis $2,3 \%$ ). Die restlichen $10 \%$ umfassen alle übrigen in diesen Höhenstufen gefund-

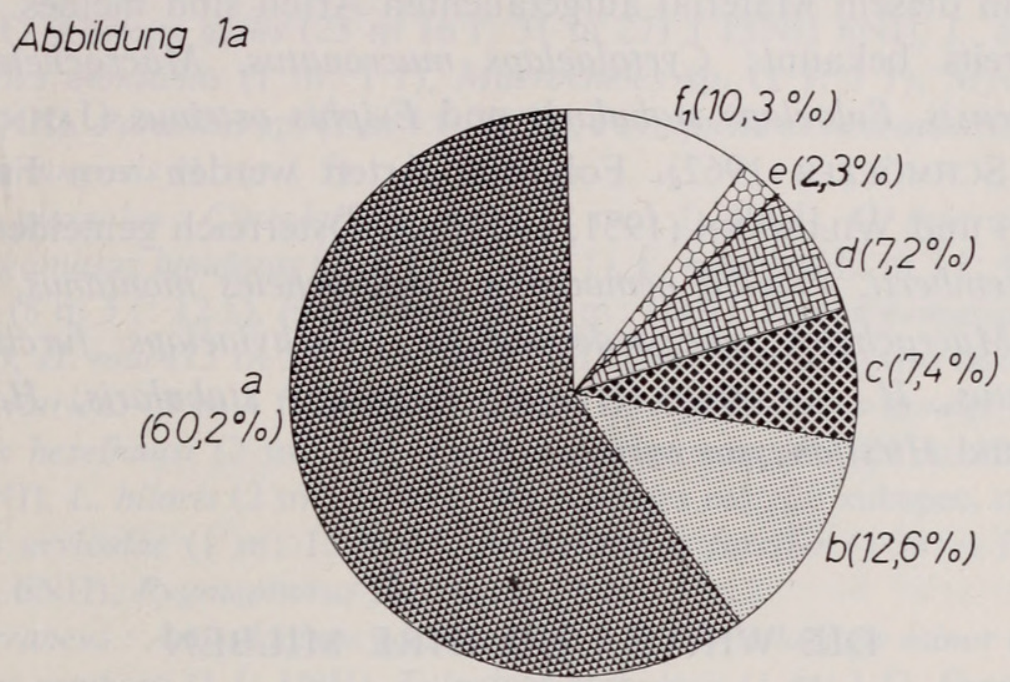

Abbildung ib

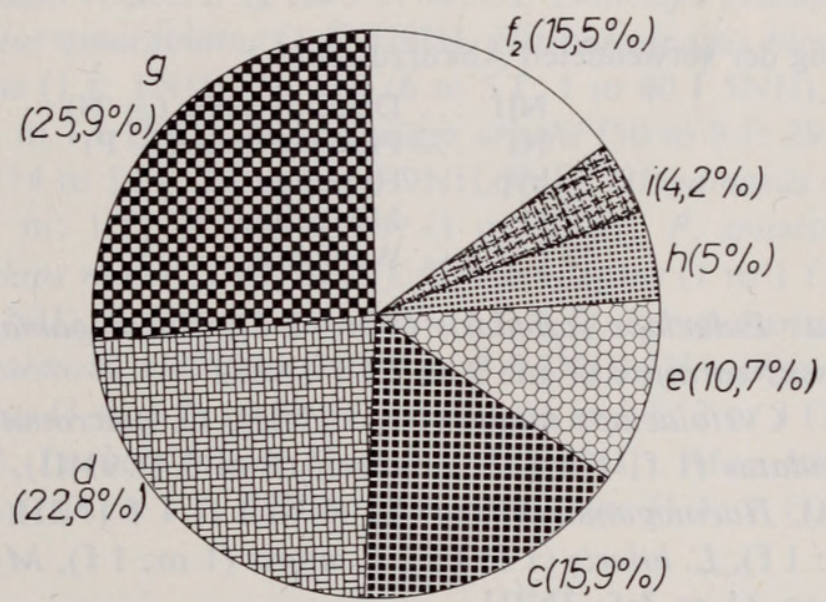

Авв. 1.

Prozentanteil der einzelnen Milbenarten (Parasitiformes vorw.) an der Gesamtausbeute aus dem Untersuchungsgebiet.

1a: Ausbeute aus der subalpinen-alpinen Stufe;

1b: Ausbeute aus Tallagen.

$\mathrm{a}=$ Laelaps clethrionomydis, $\mathrm{b}=$ Hirstionyssus tatricus, $\mathrm{c}=$ Haemogamasus nidi, $\mathrm{d}=$ Laelaps hilaris, $\mathrm{e}=$ Hyperlaelaps arvalis, $\mathrm{f}^{1}=$ restliche Arten $(18 \mathrm{spp}),. \mathrm{f}^{2}=$ restliche Arten (27 spp.), $\mathrm{g}=$ Laelaps agilis, $\mathrm{h}=$ Hirstionyssus carnifex, $\mathrm{i}=$ Eulaelaps stabularis.

enen Arten. (Abb. 1a). In den tieferen Lagen scheinen mehrere Arten auf, die ungefähr gleich stark vertreten sind: Laelaps agilis $(25,9 \%)$, L. hilaris $(22,8 \%)$, Haemogamasus nidi $(15,9 \%)$, Hyperlaelaps arvalis $(10,7 \%)$, Hirstionyssus carnifex 
$(5 \%)$, Eelaelaps stabularis $(4,2 \%)$. Ungefähr $15 \%$ entfallen auf die übrigen 2 Arten (Abb. 1b).

Von den in diesem Material aufgetauchten Arten sind meines Wissens fün aus Tirol bereits bekannt: Cyrtolaelaps mucronatus, Macrocheles montanus Veigaia nemorensis, Eulaelaps stabularis und Eviphis ostrinus (JAHN 1960, 1967 MoHr 1938; SCHMÖLzer 1962). Folgende Arten werden von Franz (1954) MrCiAK (1959) und WiLlmanN $(1951,1953)$ aus Österreich gemeldet: Eugamasu. lunulatus, E. remberti, Veigaia nemorensis, Macrocheles montanus, Cyrtolaelap. mucronatus, Macrocheles muscaedomesticae, Pachylaelaps furcifer, Haemo gamasus hirsutus, H. horridus, H. nidi, Eulaelaps stabularis, Haemogamasu: hirsutosimilis und Hirstionyssus carnifex.

\section{DIE WIRTE UND IHRE MILBEN}

Die einzelnen Milbenarten werden in alphabetischer Reihenfolge angeführt: die erste Zahlengruppe in der Klammer gibt die Anzahl der Wirte an, auf denen die betreffende Milbenart gefunden wurde, nach dem Doppelpunkt in der Klammer ist die Anzahl der auf diesem Wirt insgesamt angetroffenen Milben erwähnt. ${ }^{1}$

${ }^{1}$ Erklärung der verwendeten Abkürzungen:

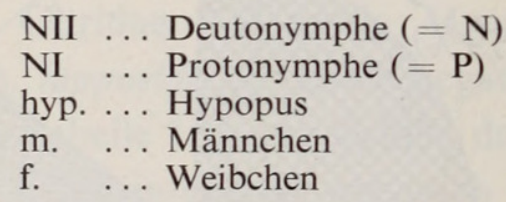

Talpa europaea: Eulaelaps stabularis (1 m: $2 \mathrm{f})$, Haemogamasus hirsutus (1 m: 1 NII), Hirstionyssus carnifex (1 m: $2 \mathrm{~m} 62 \mathrm{f} 1 \mathrm{NII})$.

Sorex araneus: Cyrtolaelaps minor (1 m: 1NII), C. mucronatus $(2 \mathrm{~m} 1 \mathrm{f}: 3 \mathrm{NII})$, Eugamasus lunulatus $(1 \mathrm{f}: 1 \mathrm{~m})$, E. remberti $(5 \mathrm{~m} 3 \mathrm{f}$ : 9NII), Euryparasitus emarginatus (1 f: 1NII), Haemogamasus horridus (1 $\mathrm{m} 1 \mathrm{f}: 1 \mathrm{f} 1 \mathrm{NII}), H$. nidi $(1 \mathrm{f}: 1 \mathrm{f})$, Laelaps agilis (1 f: $1 \mathrm{f})$, L. hilaris $(1 \mathrm{f}: 1 \mathrm{f})$, L. muris (1 $\mathrm{m}: 1 \mathrm{f})$, Myonyssus ingricus (2 f: $2 \mathrm{f})$, Parasitus sp. (1 m 2 f: 3NII).

Sorex alpinus: Eugamasus remberti (2 m: 8NII), Euryparasitus emarginatus (1 m: 1NII), Haemogamasus horridus (1 f: 1NII), Hirstionyssus eusoricis (1 m: $3 \mathrm{f})$, Parasitus sp. (1 m: 1NII), Protomyobia claparedei (2 m: $4 \mathrm{f})$, Pygmephorus spinosus (1 m: $1 \mathrm{f})$.

Neomys anomalus milleri : Eugamasus remberti (1 f: $3 \mathrm{NII})$, Haemogamasus hirsutosimilis (1 f: $1 \mathrm{f})$, Macrocheles muscaedomesticae (1 m: $1 \mathrm{f})$, Parasitus sp. (1 m: 16NII).

Nyctalus noctula: Cyrtolaelaps mucronatus $(1 \mathrm{~m}$ : $1 \mathrm{NII})$, Ichoronyssus flavus $(3 \mathrm{~m}$ : $4 \mathrm{f}$ 3NII), Spinturnix acuminatus (3 m: $3 \mathrm{~m}$ 5f), Steatonyssus spinosus (1 m: $1 \mathrm{f})$.

Myotis oxygnathus: Hirstionyssus sp. $(1 \mathrm{f}: 1 \mathrm{~m})$, Spinolaelaps jacksoni $(1 \mathrm{f}: 2 \mathrm{f})$, Spinturnix myoti (1 f: $5 \mathrm{~m} 4 \mathrm{f} \mathrm{5N}$ ), Steatonyssus spinosus (1 f: $2 \mathrm{f} \mathrm{4P),} \mathrm{Spinturnix} \mathrm{sp.}$ (1 $\mathrm{m}: 3 \mathrm{f})$.

Apodemus sp.: Cyrtolaelaps minor (4 m $1 \mathrm{f}: 5 \mathrm{NII})$, C. mucronatus (1 m $1 \mathrm{f}: 2 \mathrm{NII})$, Eugamasus lunulatus $(1 \mathrm{m:} 1 \mathrm{f})$, E. remberti $(7 \mathrm{~m} 1 \mathrm{f}: 10 \mathrm{NII})$, Eulaelaps stabularis $(10 \mathrm{~m} 5 \mathrm{f}$ : 
$2 \mathrm{~m} 24 \mathrm{f} \mathrm{1NII),} \mathrm{Euryparasitus} \mathrm{emarginatus} \mathrm{(6} \mathrm{m} 5 \mathrm{f}:$ 14NII), Haemogamasus hirsutosimilis $(2 \mathrm{~m} 5 \mathrm{f:} 26 \mathrm{f}), H$. hirsutus $(3 \mathrm{~m} 1 \mathrm{f}: 6 \mathrm{NII}), H$. horridus $(4 \mathrm{~m} 2 \mathrm{f}: 1 \mathrm{~m} \mathrm{6NII})$, H. nidi ( $8 \mathrm{~m} 8 \mathrm{f}: 1 \mathrm{~m} 118 \mathrm{f} 4 \mathrm{NII})$, Hirstionyssus isabellinus $(1 \mathrm{f}: 1 \mathrm{f}), H$. musculi (4 m: $11 \mathrm{f})$, Laelaps agilis (23 m $16 \mathrm{f}: 31 \mathrm{~m} 271 \mathrm{f} 15 \mathrm{NII} 8 \mathrm{NI})$, L. hilaris (1 f: $2 \mathrm{f})$, Macrocheles montanus (1 m: $1 \mathrm{f})$, Macrocheles sp. (1 f: $1 \mathrm{f})$, Myonyssus rossicus (1 f: $5 \mathrm{f} 1 \mathrm{NII})$, Parasitus sp. (1 m $3 \mathrm{f}: 7 \mathrm{NII})$, Poecilochirus necrophori (1 m $1 \mathrm{f}: 3 \mathrm{NII})$, Veigaia nemorensis $(1 \mathrm{~m}: 1 \mathrm{f})$.

Clethrionomys glareolus: Cyrtolaelaps minor ( $2 \mathrm{~m} 1 \mathrm{f}$ : $4 \mathrm{NII})$, C. mucronatus $(3 \mathrm{~m} 1 \mathrm{f}$ : 5NII), Eugamasus lunulatus ( $2 \mathrm{~m} 1 \mathrm{f}: 2 \mathrm{~m} 2 \mathrm{f})$, E. remberti $(2 \mathrm{~m} 2 \mathrm{f}: 4 \mathrm{NII})$, Eulaelaps stabularis ( $8 \mathrm{~m} 3 \mathrm{f}: 12 \mathrm{f})$, Eugamasus $\mathrm{sp}$. (1 m: 1NII), Haemogamasus horridus $(2 \mathrm{~m}$ : $1 \mathrm{~m} 3 \mathrm{NII}), H$. nidi (13 m $5 \mathrm{f}: 21 \mathrm{~m} 46 \mathrm{f} 7 \mathrm{NII} 1 \mathrm{NI})$, Hirstionyssus isabellinus (3 $\mathrm{m} 1 \mathrm{f}$ : $11 \mathrm{f}), H$. tatricus ( $3 \mathrm{~m} 4 \mathrm{f}: 18 \mathrm{~m} 85 \mathrm{f} \mathrm{12NII),} \mathrm{Hyperlaelaps} \mathrm{arvalis}(1 \mathrm{~m} 1 \mathrm{f}: 2 \mathrm{~m})$, Hypoaspis heselhausi $(2 \mathrm{~m}: 2 \mathrm{f})$, Laelaps clethrionomydis $(21 \mathrm{~m} 12 \mathrm{f}: 67 \mathrm{~m} 474 \mathrm{f}$ 36 NII 1NI), L. hilaris (2 m $1 \mathrm{f}: 4 \mathrm{f})$, Macrocheles matrius subspec. nov.? (1 m: $1 \mathrm{f})$, Myacarus arvicolae (1 m: 15 hyp.), Pachylaelaps furcifer (1 m: $1 \mathrm{f})$, Parasitus sp. (1 m $5 \mathrm{f}: 6 \mathrm{NII})$, Pygmephorus forcipatus (1 f: $1 \mathrm{f})$.

'itymys subterraneus: Androlaelaps sardous (1 f: $3 \mathrm{f})$, Cyrtolaelaps minor (1 $\mathrm{m} 1 \mathrm{f}: 2 \mathrm{NII})$, Eugamasus remberti (1 f: 1NII), Eulaelaps stabularis ( $1 \mathrm{~m}: 1 \mathrm{f})$, Euryparasitus emarginatus (1 f: 1NII), Eviphis ostrinus (1 f: $1 \mathrm{f})$, Haemogamasus horridus (2 f: 2NII), H. nidi (1 m $1 \mathrm{f}: 2 \mathrm{~m} 7 \mathrm{f} 1 \mathrm{NII})$, Hirstionyssus isabellinus $(1 \mathrm{f}: 1 \mathrm{f})$, Laelaps hilaris (1 m: $1 \mathrm{f})$, Macrocheles matrius subspec. nov.? ( $2 \mathrm{~m}: 2 \mathrm{f})$, Parasitus sp. (1 m: 1NII). Microtus arvalis rufescentefuscus : Haemogamasus nidi $(1 \mathrm{f}: 1 \mathrm{~m} 1 \mathrm{f})$, Hyperlaelaps arvalis ( $2 \mathrm{f}: 6 \mathrm{~m} 12 \mathrm{f} 1 \mathrm{NI})$, Laelaps hilaris $(1 \mathrm{f}: 22 \mathrm{f})$.

Microtus agrestis: Eugamasus remberti $(2 \mathrm{~m} 3 \mathrm{f}$ : $6 \mathrm{NII})$, Eulaelaps stabularis $(7 \mathrm{~m} 2 \mathrm{f}$ : $2 \mathrm{~m} 14 \mathrm{f})$, Euryparasitus emarginatus (1 f: 1NII), Haemogamasus hirsutus (1 m $1 \mathrm{f}$ : $1 \mathrm{~m} \mathrm{1NII}), H$. horridus (1 f: $1 \mathrm{NII}), H$. nidi $(6 \mathrm{~m} 5 \mathrm{f}: 1 \mathrm{~m} 40 \mathrm{f} 5 \mathrm{NII})$, Hirstionyssus isabellinus ( $3 \mathrm{~m} 3 \mathrm{f}: 2 \mathrm{~m} 7 \mathrm{f} 1 \mathrm{NII})$, Hyperlaelaps arvalis $(10 \mathrm{~m} 9 \mathrm{f}: 29 \mathrm{~m} 82 \mathrm{f} 12 \mathrm{NII}$ 2NI), Laelaps hilaris (14 m $12 \mathrm{f}: 24 \mathrm{~m} 231 \mathrm{f} 9 \mathrm{NII} 3 \mathrm{NI})$, Ologamasus sp. (1 m: $1 \mathrm{f})$, Veigaia nemorensis $(1 \mathrm{~m}: 1 \mathrm{f}), P$. necrophori $(1 \mathrm{~m}$ : 2NII), P. spinosus $(1 \mathrm{~m}: 1 \mathrm{f})$. Microtus nivalis: Cyrtolaelaps minor (1 m: $1 \mathrm{NII})$, C. mucronatus $(1 \mathrm{~m} 1 \mathrm{f}: 2 \mathrm{NII})$, Eugamasus remberti (1 f: $1 \mathrm{NII})$, Eulaelaps stabularis (1 f: $1 \mathrm{f})$, Euryparasitus emarginatus (1 m: 1NII), Haemogamasus nidi $(2 \mathrm{~m} 3 \mathrm{f}: 7 \mathrm{~m} 8 \mathrm{f} 3 \mathrm{NII})$, H. nidiformis (1 f: $2 \mathrm{f})$, Hirstionyssus isabellinus (1 $\mathrm{m} 4 \mathrm{f}: 10 \mathrm{f}), H$. tatricus $(3 \mathrm{~m} 1 \mathrm{f}: 2 \mathrm{~m} 3 \mathrm{f})$, Hyperaelaps arvalis $(1 \mathrm{f}: 1 \mathrm{~m} 1 \mathrm{f})$, Laelaps hilaris $(7 \mathrm{~m} 5 \mathrm{f}: 41 \mathrm{f} 1 \mathrm{NII})$, Macrocheles montanus (1 f: 1 f), Parasitus sp. (2 $\mathrm{m} 1 \mathrm{f}$ : 5NII), Pergamasus sp. (1 f: $1 \mathrm{f})$, Proctolaelaps pygmaeus $(1 \mathrm{~m}: 2 \mathrm{f})$.

\section{BESPRECHUNG DER ARTEN}

Folgende Trombiculiden-Arten wurden nachgewiesen, sie können aufgrund der unregelmässigen Aufsammlungen nur kurz behandelt werden und stellen sicherlich nur die in Tirol häufigsten Arten dar: Leptotrombidium intermedia europaea DANIEL et BERLIH, Leptotrombidium komareki DANIEL et DUSBABEK, Neotrombicula autumnalis autumnalis (RICHARDS), Neotrombicula autumnalis inopinatum (Oudemans), Neotrombicula autumnalis earis KePKA, Neotrombicula 
zachvatkini (SCHLUGER) und Trombicula toldti WINKLER; Leptotrombidium komareki konnte erstmals für Tirol nachgewiesen werden, als neuer Wirt schein Nyctalus noctula auf. Ebenfalls neue Wirte konnten für L. intermedia europaec (Myotis oxygnathus) und T. toldti (Neomys anomalus) gefunden werden.

Die systematische Reihung der nachstehenden parasitischen Acari wurde grossteils nach Evans (1957) vorgenommen.

\section{Protomyobia claparedei (Poppe, 1896)}

Obergurgl, $1900 \mathrm{~m}$, Sorex alpinus : $4 \mathrm{f}$ (Fangmonat: VI).

Nach Willmann (1952) ein typischer Parasit von S. araneus; Myobien sind im allgemeinen sehr wirtstreu und auf bestimmte Säuger spezialisiert.

\section{Pygmephorus spinosus (Kramer, 1877)}

Umgebung Innsbruck, 800 m, Microtus agrestis : $1 \mathrm{f}$ (IV) - Obergurgl 1900 m, Sorex alpinus : $1 \mathrm{f}(\mathrm{VI})$.

Pygmephorus forcipatus Willmann, 1952

Obergurgl, $2000 \mathrm{~m}$, Clethrionomys glareolus : $1 \mathrm{f}$ (VI).

\section{Pergamasus sp.}

Timmelsjoch (Obergurgl), $2500 \mathrm{~m}$, Microtus nivalis : $1 \mathrm{f}$ (VIII).

Eugamasus lunulatus (J. Müller, 1859)

Umgebung Innsbruck, bis $1000 \mathrm{~m}$, Sorex araneus, Apodemus flavicollis, Clethrionomys glareolus : $3 \mathrm{~m} 3 \mathrm{f}$ (II, VI, VII, IX).

\section{Eugamasus remberti Oudemans, 1912}

Umgebung Innsbruck, Sorex araneus, Neomys anomalus, Apodemus flavicollis, Microtus agrestis: 26NII. (II-VII, IX, XII) - Obergurgl, 1900-2100 m, Sorex alpinus, $S$. araneus, Pitymys subterraneus, Clethrionomys glareolus : 16NII. (III, V-VII) Kühtai, 2400 m, Microtus nivalis 1NII (VII).

Die adulten Stadien treten in den Nestern der Wirte auf, im Fell werden fast ausnahmslos die Deutonymphen angetroffen (MrCIAK 1959, MrCIAK et BRANDER 1967, MrCIAK et al. 1966). 


\section{Eugamasus sp.}

Umgebung Innsbruck, Clethrionomys glareolus : 1NII (VI).

\section{Parasitus sp.}

Umgebung Innsbruck, Sorex alpinus, S. araneus, Neomys anomalus, Apodemus flavicollis, A. sylvaticus : 26NII (III, VI-X) - Obergurgl, 1800-2100 m, Sorex araneus, Pitymys subterraneus, Clethrionomys glareolus, Microtus nivalis : 13NII (VI-IX).

\section{Poecilochirus necrophori Vitzthum, 1930}

Umgebung Innsbruck, Apodemus sylvaticus, Apodemus sp., Microtus agrestis: 5 NII (I, VI, VIII).

Die Art kommt nur zufällig auf Kleinsäugern vor, normal parasitiert sie Käfer der Gattungen Geotrupes und Necrophorus (MRCIAK 1959, MrCIAK et BRANDER 1965); MoHr (1954) erwähnt diese Art als Parasit von A. flavicollis, A. sylvaticus, C. glareolus und $M$. agrestis.

\section{Cyrtolaelaps minor Willmann, 1952}

Umgebung Innsbruck, bis $1400 \mathrm{~m}$, Sorex araneus, Apodemus flavicollis, Clethrionomys glareolus : 9NII (IV-VIII, XI) - Obergurgl, 1900-2100 m, Pitymys subterraneus, Clethrionomys glareolus, Microtus nivalis : 3NII (VI, VII) - Hinterbärenbad (Kaisergebirge), $850 \mathrm{~m}$, Pitymys subterraneus : 1NII (VI).

Im Fell treten die Deutonymphen auf, das Vorkommen auf Kleinsäugern ist jedoch selten und zufällig (MrCIAK 1959).

\section{Cyrtolaelaps mucronatus (G. et R. Canestrini, 1881)}

Umgebung Innsbruck, bis $1000 \mathrm{~m}$, Nyctalus noctula, Sorex araneus, Apodemus flavicollis, Clethrionomys glareolus: 8NII (I, IV, VIII, IX, XI) - Obergurgl, 1900-2500 m, Sorex araneus, Clethrionomys glareolus, Microtus nivalis : 9NII (IV-VI, VIII, IX) Kühtai, 2250 m, Microtus nivalis : 8NII (IX).

Der Fund auf $N$. noctula ist überraschend, denn bislang war die Art nur von Nagern und Spitzmäusen bekannt (EDLER 1969, MrCiAK et TovorniK 1966); Franz (1954) kennt die Art (sub Protolaelaps mucronatus) von mehreren Fundorten, darunter auch vom Türkenloch bei Kleinzell, einem bekannten Fledermausquartier. Willmann (1952) fand sie zweimal in Murmeltiernestern in der Steiermark. 
Euryparasitus emarginatus (C. L. Koch, 1839)

Umgebung Innsbruck, bis $1400 \mathrm{~m}$, Sorex araneus, Apodemus flavicollis, A. sylvaticus Microtus agrestis : 16NII (I, IV-VI, VIII, IX, XI, XII) - Obergurgl, 1900-2100 m Sorex alpinus, Pitymys subterraneus, Microtus nivalis: 3NII (VI, VII) - Hinter. bärenbad (Kaisergebirge), $850 \mathrm{~m}$, Pitymys subterraneus : 1NII (VI).

Diese weit verbreitete Art lebt frei und räuberisch, kommt jedoch oft in Kleinsäugernestern und auch im Fell der Tiere selbst vor (EDLER 1969).

\section{Ologamasus sp.}

Umgebung Innsbruck, Microtus agrestis : $1 \mathrm{f}$ (VI).

Macrocheles matrius subspec. nov.?

Obergurgl, 2000-2100 m, Pitymys subterraneus, Clethrionomys glareolus : $3 \mathrm{f}$ (VII).

Nach MrciaK (in litt.) könnte es sich hier um eine neue Unterart handeln. Das Vorkommen der Art im Haarkleid der Säuger ist mehr zufällig (MrCIAK 1959).

Macrocheles montanus (Willmann, 1951)

Umgebung Innsbruck, Apodemus flavicollis: 1 f (VIII) - Kühtai, 2300 m, Microtus nivalis : $1 \mathrm{f}(\mathrm{V})$.

Macrocheles muscaedomesticae (Scopoli, 1772)

Umgebung Innsbruck, Neomys anomalus : $1 \mathrm{f}$ (III).

\section{Macrocheles sp.}

Umgebung Innsbruck, Apodemus sylvaticus : $1 \mathrm{f}$ (VIII).

Proctolaelaps pygmaeus (J. Müller, 1859)

Obergurgl, $2000 \mathrm{~m}$, Microtus nivalis : $2 \mathrm{f}(\mathrm{V})$.

Die Art kommt hauptsichtlich in abgelagerten Pflanzenresten vor, weshalb die Nester verschiedener Kleinsäuger günstige Lebensbedingungen für sie bieten (MRCIAK et BRANDER 1965). 
Veigaia nemorensis (C. L. Koch, 1839)

Umgebung Innsbruck, Apodemus flavicollis, Microtus agrestis : $2 \mathrm{f}$ (VI).

JAHN (1967) konnte die Art von mehreren Fundorten Nordtirols aus Bodenproben gewinnen.

Pachylaelaps furcifer Oudemans, 1903

Umgebung Innsbruck, $1000 \mathrm{~m}$, Clethrionomys glareolus : $1 \mathrm{f}$ (VIII).

Diese normal freilebende räuberische Art kommt nur zufällig auf Kleinsäugern vor (MRCIAK et TOVORNIK 1966).

Eviphis ostrinus (C. L. Koch, 1836)

Hinterbärenbad (Kaisergebirge), Pitymys subterraneus : $1 \mathrm{f}$ (VI).

Die Art steigt in den Alpen bis zur Obergrenze der Zwergstrauchheidenstufe empor (FRANZ 1954).

Hypoaspis heselhausi Oudemans, 1912

Umgebung Innsbruck, $1000 \mathrm{~m}$, Clethrionomys glareolus : 2 f (VI, VII).

Androlaelaps sardous Berlese, 1911

Hinterbärenbad (Kaisergebirge), $850 \mathrm{~m}$, Pitymys subterraneus : $3 \mathrm{f}$ (VI).

Das Vorkommen auf den Wirten und auch in den Nestern dürfte eher selten sein; MrсiAK et al. (1966) fanden die Art am zahlreichsten in den Nestern der Rötelmaus C. glareolus.

\section{Eulaelaps stabularis (C. L. Koch, 1836)}

Umgebung Innsbruck, bis $1000 \mathrm{~m}$, Talpa europaea, Apodemus flavicollis, A. sylvaticus, Clethrionomys glareolus, Microtus agrestis : $48 \mathrm{f} 6 \mathrm{~m}$ 1NII (I, III-XI) - Obergurgl, 2000-2100 m, Pitymys subterraneus, Clethrionomys glareolus : $4 \mathrm{f}$ (V-VII) - Kühtai, $2400 \mathrm{~m}$, Microtus nivalis : $1 \mathrm{f}$ (VII).

Das Wirtsspektrum dieser Art ist sehr gross, sie bewohnt die Nester fast aller Kleinsäugerarten, wobei es manchmal fast zu einer Versuchung der Nester kommen kann. E. stabularis stellt, nach Untersuchungen von DANIEL (1969), aktiv zu den Nestern wandernde Art dar, die keine allzu feste Beziehungen zur Umgebung aufweist und deshalb oberirdische wie unterirdische Nester besiedelt, 
während z.B. $H$. nidi deutliche Abhängigkeit von mikroklimatischen Faktoren aufweist und daher oberirdische Nester bevorzugt. Sie kann auch den Menschen befallen (MrCIAK 1959); die Art ist vom Patscherkofel bei Innsbruck auf $M$. nivalis gefunden worden (MOHR 1938).

\section{Laelaps agilis C. L. Koch, 1836}

Umgebung Innsbruck, bis $1400 \mathrm{~m}$, Sorex araneus, Apodemus flavicollis, A. sylvaticus, Apodemus sp.: $31 \mathrm{~m} 272 \mathrm{f} 15 \mathrm{NII} 8 \mathrm{NI}$.

Das Auftreten dieser für die Gattung Apodemus typischen Art auf S. araneus ist ungewöhnlich und kommt normalerweise nur bei sehr engem Kontakt zwischen Waldspitzmaus und Apodemus spp. vor. Die Deutonymphen tauchten in den Monaten I, II, V, VI und XI auf, Protonymphen konnten auf den Wirten nur in den Monaten April bis Juni gefunden werden. (Tabelle 2).

\section{TABELLE 2}

Auftreten von L. agilis auf Apodemus spp. in den einzelnen Monaten, angegeben ist die Exemplarzahl pro Wirt.

\begin{tabular}{|c|c|c|c|c|c|c|c|c|c|c|c|c|}
\hline & I & II & III & IV & $\mathrm{V}$ & VI & VII & VIII & IX & $\mathrm{x}$ & XI & XII \\
\hline $\mathrm{m}$ & 0,3 & 0,2 & - & 0,3 & 1,5 & 0,1 & 1,0 & - & 0,1 & - & 0,2 & - \\
\hline f & 5,8 & 2,7 & 0,2 & 5,9 & 7,5 & 1,4 & 4,0 & 1,8 & 4,1 & 1,3 & 2,7 & - \\
\hline NII & 0,1 & 0,2 & - & - & 1,0 & 0,2 & - & - & - & - & 0,1 & - \\
\hline NI & - & - & - & 0,5 & 0,3 & 0,1 & - & - & - & - & - & - \\
\hline unters. Apod. & 11 & 10 & 5 & 7 & 7 & 11 & 7 & 11 & 11 & 6 & 8 & 4 \\
\hline
\end{tabular}

Es hat demnach den Anschein, als ob neben den Adulti auch Deutonymphen überwintern und im Frühjahr zu Adulten heranwachsen, die Eiablage dürfte im frühen Frühjahr erfolgen, sodass die Protonymphen ungefähr im April auftauchen. Die im April und Mai auftretenden Deutonymphen könnten bereits aus diesjährigen NI entstanden sei. Das (allerdings einzige) darauf hin untersuchte Material wurde Ende Jänner bis Anfang Feber gesammelt: in dieser Zeit konnten 4 Männchen 47 Weibchen 3NII festgestellt werden. Von diesen Weibchen waren 7 Weibchen ohne Eier, 5 Weibchen befanden sich am Anfang der Ovulation, 24 Weibchen trugen Eier, 10 Weibchen zeigten Larven, ein Weibchen hatte sich eben entpuppt (MrCiaK, in litt.). Im Untersuchungsgebiet scheint die Hauptvermehrunszeit dieser Art, nach dem Auftreten von Protonymphen zu schliessen, im späten Frühjahr zu liegen; die Zahl der Milben pro Wirt erreicht in den Monaten April und Mai das Maximum. Männchen konnten nur selten und 


\section{Abbildung 2 a}

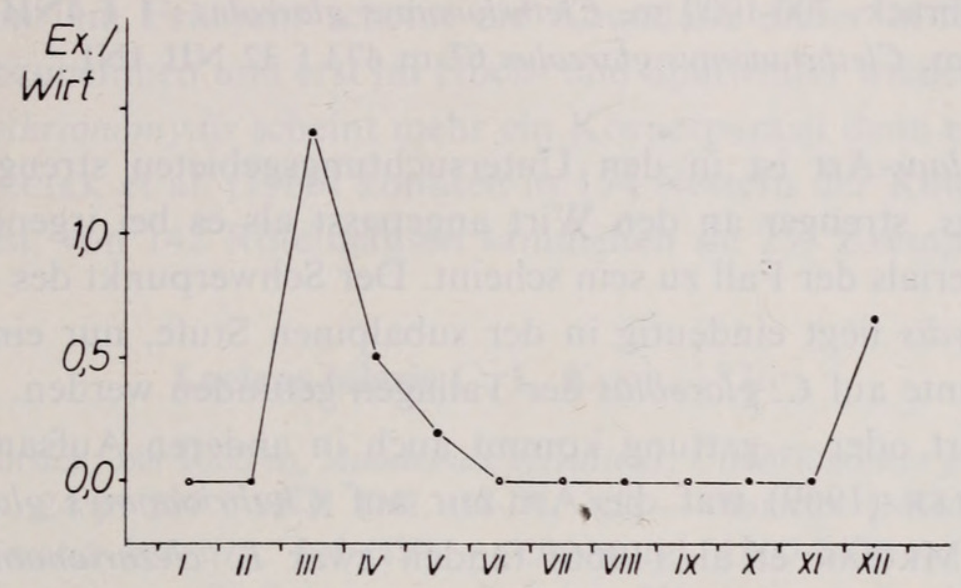

Abbildung $2 b$

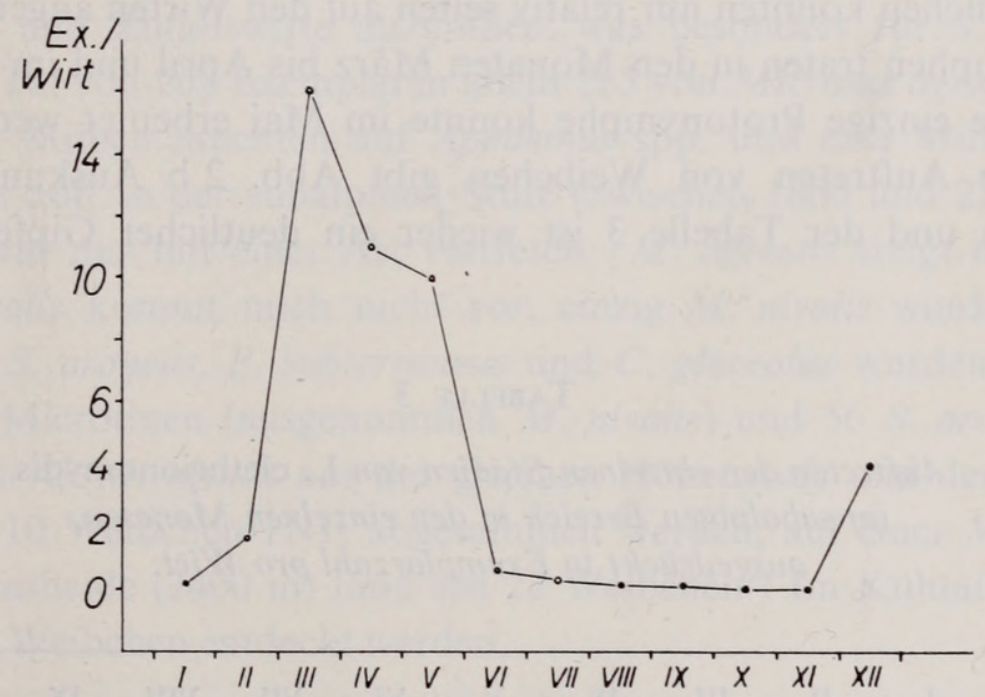

AвB. 2.

Jahreszeitliche Schwankungen im Auftreten zweier Stadien von Laelaps clethrionomydis auf Clethrionomys glareolus der subalpinen Stufe des Untersuchungsgebietes.

2a: Laelaps clethrionomydis: NII (Deutonymphen);

2b: Laelaps clethrionomydis: Weibchen.

poradisch auf den Wirten gefunden werden. KARG (1961) vermutete bei seinen Jntersuchungen an Dendrolaelaps rectus, dass die meisten Weibchen noch vor lem Winter befruchtet werden; das Auftreten von Larven und Nymphen zeigte in deutliches Frühjahrs- u. Sommermaximum. 


\section{Laelaps clethrionomydis Lange, 1955}

Umgebung Innsbruck, 700-1000 m, Clethrionomys glareolus : 1 f 4NII. - Obergurgl 1800-2100 m, Clethrionomys glareolus 67 m 473 f 32 NII 1NI.

Diese Laelaps-Art ist in den Untersuchtungsgebieten streng wirtspezifisch auf C. glareolus, strenger an den Wirt angepasst als es bei irgendeiner anderer Art dieses Materials der Fall zu sein scheint. Der Schwerpunkt des Auftretens vor L. clethrionomydis liegt eindeutig in der subalpinen Stufe, nur ein Bruchteil de Exemplare konnte auf $C$. glareolus der Tallagen gefunden werden. Diese Bindun an eine Wirtsart oder - gattung kommt auch in anderen Aufsammlungen zun Ausdruck: EDLER (1969) traf die Art nur auf Clethrionomys glareolus und $C$ rufocanus an. MrCiAK et al. (1966) fanden zwar L. clethrionomydis auch au $S$. araneus, A. flavicollis, A. sylvaticus und P. subterraneus, hauptsächlich erwie: sich aber $C$. glareolus befallen. Als südwestliche Grenze der Verbreitung vor L. clethrionomydis nimmt MrCIAK (1959) das Gebiet der Steiner Alpen (Jugo. slawien) an, Fundorte liegen aus Mitteleuropa, Ost- und Nordeuropa und aus Russland vor (MRCIAK 1959, EdLER 1969).

Die Männchen konnten nur relativ selten auf den Wirten angetroffen werden die Deutonymphen traten in den Monaten März bis April und im Dezember auf (Abb. 2a), die einzige Protonymphe konnte im Mai erbeutet werden. Über das jahreszeitliche Auftreten von Weibchen gibt Abb. 2 b Auskunft. Aus dieser Darstellungen und der Tabelle 3 ist wieder ein deutlicher Gipfel im Frühjahı ersichtlich.

\section{TABELLE 3}

Auftreten der einzelnen Stadien von L. clethrionomydis im subalpinen Bereich in den einzelnen Monaten, ausgedrückt in Exemplarzahl pro Wirt.

\begin{tabular}{lccccccccccccc}
\hline & I & II & III & IV & V & VI & VII & VIII & IX & X & XI & XI \\
\hline m & - & 0,8 & 2,2 & 0,4 & 0,1 & - & 0,3 & - & - & - & - & 2 \\
f & 0,1 & 1,6 & 16 & 11 & 10 & 0,6 & 0,7 & 0,1 & 0,01 & - & - & 4 \\
NII & - & - & 1,4 & 0,2 & 0,2 & - & - & - & - & - & - & 1 \\
NI & - & - & - & - & 0,1 & - & - & - & - & - & - & - \\
\hline
\end{tabular}

Das völlige Fehlen von L. clethrionomydis in den Monaten Oktober und November ist auffällig und unerklärlich: es wurden 13 bzw. 10 C. glareolus in den selben Biotopen wie z.B. 11 C. glareolus im März untersucht; im April wurden gleich viele $C$. glareolus (10) wie im November untersucht, es konnten 116 Exemp- 
are dieser Milbenart gefunden werden. Auffällig auch der abrupte Gipfel im Jezember: von 14 C. glareolus wurden 109 L. clethrionomydis abgesammelt. Nach iner „Explosion“ im Frühjahr scheint die Abundanz dieser Milbenart auf den Virten stetig abzunehmen und erst im Hoch- und Spätwinter wieder zuzunehmen.

Laelaps clethrionomydis scheint mehr ein Körperparasit denn ein Nestbewoher zu sein: MrCiAK et al. (1966) konnten in 154 Nestern der Rötelmaus nur ein Veibchen finden, von 142 Rötelmäusen sammelten sie 235 Exemplare ab.

\section{Laelaps hilaris C. L. Koch, 1836}

Jmgebung Innsbruck, bis $1000 \mathrm{~m}$, Apodemus sylvaticus, Clethrionomys glareolus, Microtus agrestis: $24 \mathrm{~m} 229 \mathrm{f}$ 9NII 1NI. (II-IX, XI) - Barwies (Mieminger Plateau), $800 \mathrm{~m}$, Microtus agrestis : $5 \mathrm{f}$ (IV) - Obergurgl, 1800-2400 m, Sorex araneus, Pitymys subterraneus, Clethrionomys glareolus, Microtus arvalis, Microtus nivalis :

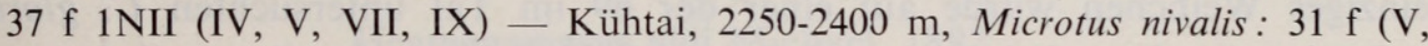
VIII, IX).

L. hilaris scheint im Untersuchungsgebiet eine gewisse Bindung an die Jattung Microtus aufzuweisen, die übrigen Kleinsäugerarten sind eher als Jelegenheits - und Zufallswirte anzusehen, was besonders für $S$. araneus gilt. io konnten im Tal von 268 Exemplaren allein 265 von Microtus agrestis abgesamnelt werden, 2 Milben tauchten auf Apodemus spp. und eine Milbe dieser Art uf $C$. glareolus auf. In der subalpinen Stufe (zwischen 1800 und $2200 \mathrm{~m}$ ) ist die Jattung Microtus nur mit einer Art vertreten ( $M$. agrestis steigt nicht so hoch inauf, $M$. arvalis kommt noch nicht vor, einzig $M$. nivalis wurde regelmässig efangen); auf $S$. araneus, $P$. subterraneus und $C$. glareolus wurden 5 Weibchen ;efunden: 140 Microtinen (ausgenommen $M$. nivalis) und $56 \mathrm{~S}$. araneus wurden Intersucht. Von 43 M. nivalis aus der gleichen Höhenstufe und den selben Bioopen konnten 10 Weibchen 1 NII abgesammelt werden, auf einei $M$. arvalis aus ler alpinen Grasheide $(2400 \mathrm{~m})$ fand ich 22 Weibchen ! Im Kühtai konnten auf 3 M. nivalis 31 Weibchen entdeckt werden.

Laelaps muris (Ljunh, 1799)

Jmgebung Innsbruck, $1000 \mathrm{~m}$, Sorex araneus : $1 \mathrm{f}$ (VIII).

Die Art wird als spezifischer Parasit der Gattung Arvicola angesehen MrCIAK et TovorniK 1966, EdLER 1969), das Vorkommen auf S. araneus ist ufällig und ist wahrscheinlich als Folge eines engen Kontaktes mit Arvicola errestris zu betrachten. 
Hyperlaelaps arvalis (Zachvatkin, 1948)

Umgebung Innsbruck, bis 1000 m, Clethrionomys glareolus, Microtus agrestis : 30 m 90 12NII 2NI (III-VII, IX). - Barwies (Mieminger Plateau), $800 \mathrm{~m}$, Microtus agrestis $1 \mathrm{f}$ (IV). - Obergurgl, 2000-2400 m, Clethrionomys glareolus, Microtus arvalis Microtus nivalis : $4 \mathrm{~m} 4 \mathrm{f}$ 1NI (III, V, IX). - Kühtai, $2300 \mathrm{~m}$, Microtus arvalis $4 \mathrm{~m} 9 \mathrm{f}(\mathrm{V})$.

M. arvalis gilt als Hauptwirt dieser Art, kommt aber auch auf andere Gattungen der Microtinen häufig vor (MrCiaK et Brander 1965, MrCiAK e TOVORNIK 1966). Im Untersuchungsgebiet zeigt $H$. arvalis eine enge Bindun: an die Erdmaus $M$. agrestis (oder an das von dieser Art bewohnte Biotop?) $29 \mathrm{~m} 90 \mathrm{f} 12 \mathrm{NII}$ 2NI konnten auf diesem Kleinsäuger festgestellt werden, eiı einziges Weibchen wurde auf einer der $69 \mathrm{im}$ Tal untersuchten C. glareolu gefunden. In der subalpinen und alpinen Stufe konnte der Grossteil des gesam melten Materials auf $M$. arvalis gefangen werden (19 Exemplare), die in de alpinen Grasheide lebt, 2 Exemplare tauchten auf insgesamt 130 untersuchte M. nivalis und ein Männchen auf 134 gefangenen C. glareolus auf ! Die klein Zahl auf $M$. nivalis überrascht da zahlreiche Tiere im gleichen Biotop wie $M$ arvalis gefangen wurden. Die Denkmöglichkeit, dass das Nest der $M$. nivali. keine allzu günstigen Entwicklungsbedingungen bietet, ist wenig wahrscheinlich Dieses „Meiden“ der Schneemaus durch H. arvalis könnte andererseits aber aucl ein ökologischer Hinweis dafür sein, dass die Schneemaus, wie LEHMAN (1969 vermutet, in eine eigene Gattung zu stellen ist und eher mit der Gattung Cle thrionomys denn mit Microtus verwandt ist.

Myonyssus ingricus Bregetova, 1956

Obergurgl, $2100 \mathrm{~m}$, Sorex araneus : $2 \mathrm{f}(\mathrm{V}, \mathrm{VIII})$.

Die Wirtsbeziehungen dieser Art sind noch unklar: obzwar die Art meis von Sorex spp. abgesammelt wird (MrCIAK et BRANDER 1965), konnte bei Nestun tersuchungen in den westlichen Karpathen die grösste Anzahl in Nestern de Rötelmaus C. glareolus gefunden werden (MrCIAK et al. 1966).

Myonyssus rossicus Bregetova, 1956

Umgebung Innsbruck, Apodemus flavicollis : 5 f 1NII (XI).

$M$. rossicus tritt, wie $M$. ingricus, auf den Wirten anscheinend recht selter auf, bei Nestuntersuchungen (MRCIAK et al., op. cit.) konnte wieder die grösste 
Zahl in Rötelmausnestern gefunden werden. Die Art wurde bisher nur aus Russand, CSSR, Bulgarien, Albanien (MrCIAK 1959, 1960) u. Rumänien bekannt.

\section{Haemogamasus horridus Michael, 1892}

Jmgebung Innsbruck, bis $1400 \mathrm{~m}$, Sorex araneus, Apodemus flavicollis, A. sylvaticus, Clethrionomys glareolus, Microtus agrestis: $2 \mathrm{~m} 1 \mathrm{f} 10 \mathrm{NII}$ (V, VI, VII, IX). Hinterbärenbad (Kaisergebirge), 800 m, Pitymis subterraneus 1NII (VI). - Obergurgl, $2100 \mathrm{~m}$, Sorex alpinus, S. araneus, Pitymys suhterraneus : 3NII (VII, VIII).

Auf dem Wirt selbst halten sich häuptsächlich die Deutonymphen auf, vährend die adulten Stadien mehr in den Nestern zu finden sind (EDLER 1969, MRCIAK et BRANDER 1965).

\section{Heamogamasus nidi Michael, 1892}

Jmgebung Inssbruck, bis $1000 \mathrm{~m}$, Sorex araneus, Apodemus flavicollis, A. sylvaticus, Apodemus sp., Clethrionomys glareolus, Microtus agrestis: $7 \mathrm{~m} 174 \mathrm{f} 14$ NII (während des ganzen Jahres). - Hinterbärenbad (Kaisergebirge) 800 m, Pitymys subterraneus: $2 \mathrm{~m} 3 \mathrm{f}$ (VI). - Obergurgl, 2000-2650 m, Pitymys subterraneus, Clethrionomys glareolus, Microtus nivalis : $24 \mathrm{~m} 44 \mathrm{f}$ 5NII 1NI (III, V, VII, VIII, IX, XI, XII). - Kühtai, $2100 \mathrm{~m}$, Microtus nivalis : 1NII (IX).

Als bevorzugter Wirt scheint in diesem Material A. flavicollis auf $(43,8 \%$ ller gesammelten Exemplare stammen von diesem Wirt, der durchschnittlich on 1,5 Milben dieser Art befallen war), der zweitgrösste Prozentanteil konnte von ¿. glareolus abgesammelt werden $(27,3 \%)$, doch wurden auf einer Rötelmaus im Jurchschnitt nur $0,3 H$. nidi angetroffen, während auf $M$. agrestis (bei einem Inteil von 20\%) 1,2 Exemplare pro Wirt gefunden wurden. Die Prozentzahlen, vie sie auch EDLER (1969) z.B. angibt, sind m.E. nach nicht geeignet, eine Aussage ber eine eventuelle Wirtsbevorzugung zu erlauben; dazu müsste die Zahl der intersuchten Wirte in Beziehung mit den Milbenzahlen gebracht werden. Ihre erbreitung erstreckt sich über die gesamte nördliche Hemisphäre (MRCIAK 1959); a Österreich stellte Franz (1954) die Art bis 2500 m fest, SchweIzer (1949) rwähnt sie aus Höhen zwischen $1900 \mathrm{~m}$ und $2300 \mathrm{~m}$. H. nidi bevorzugt, wie auch 1. hirsutus, oberirdische Nester und besiedelt diese relativ stabil (DANIEL 1969).

\section{Haemogamasus nidiformis Bregetova, 1955}

iühtai, $2250 \mathrm{~m}$, Microtus nivalis : $2 \mathrm{f}$ (IX).

Während in Mittel- und Osteuropa diese Art hauptsächlich in grösseren Iöhen vorkommt (MRCIAK 1959), tritt sie in Nordeuropa (Schweden) auch in 
Höhen zwischen $400 \mathrm{~m}$ und $800 \mathrm{~m}$ auf (EDLER 1968, 1969). Bisher ist sie nur au der USSR, aus der Tschechoslowakei, aus Bulgarien und Schweden gemelde worden. Der einzige Fund in Nordtirol gelang an der oberen Grenze der Zwerg strauchheide.

\section{Haemogamasus hirsutus Berlese, 1889}

Umgebung Innsbruck, bis $1000 \mathrm{~m}$, Talpa europaea, Apodemus flavicollis, Microtus agres tis: $1 \mathrm{~m}$ 7NII (IV-VII, XI). - Barwies (Mieminger Plateau) $800 \mathrm{~m}$, Microtu agrestis : 1NII (IV).

Im Fell des Wirtes treten nur selten die adulten Formen auf; das Wirtsspek trum ist umfangreich, Vorkommen der Art sind aus allen Teilen Europas gemelde (EDLER 1969). Die Art kann als Uberträger einer Encephalitis von medizinische Bedeutung sein (MRCIAK et BRANDER 1965).

\section{Haemogamasus hirsutosimilis Willmann, 1952}

Umgebung Innsbruck, bis $100 \mathrm{~m}$, Neomys anomalus, Apodemus flavicollis : $27 \mathrm{f}$.

Diese mit $H$. hirsutus nahe verwandte Art weist eine enge Beziehung mi Vertretern der Gattung Apodemus auf. Sie wurde von WiLlmann nach Materia von A. flavicollis und A. agrarius beschrieben, MrCIAK et TovorniK (1966) fander sie auf A. flavicollis, in den vorliegenden Material wurden 26 Weibchen vor A. flavicollis abgesammelt, nur ein Weibchen tauchte (als Zufallsfund?) au N. anomalus auf. Sie konnte in den Monaten I, II, IV, VI, XI und XII gefundeI werden.

Hirstionyssus isabellinus Oudemans, 1913

Umgebung Innsbruck, bis 1200 m, Apodemus flavicollis, Clethrionomys glareolus, Microtu agrestis : $2 \mathrm{~m} 18 \mathrm{f} 1 \mathrm{NII}$ (IV, VI-VIII). - Hinterbärenbad (Kaisergebirge), $800 \mathrm{~m}$ Pitymys subterraneus : $1 \mathrm{f}(\mathrm{VI})$. - Obergurgl, 1900-2500 m, Clethrionomys glareolus Microtus nivalis : $7 \mathrm{f}$ (VI, VIII). — Kühtai, $2250 \mathrm{~m}$, Microtus nivalis : $4 \mathrm{f}$ (VIII, IX

Die Art parasitiert vor allem auf Microtiden, kommt aber auch auf Soricides vor (EDLER 1969); sie ist aber auch auf Musteliden gefunden worden, auf die si vielleicht von den erbeuteten Kleinsäugern übergegangen ist (MrCIAK e BRANDER 1965). In Nordtirol dürfte sie eine der höchststeigenden parasitischer Milben sein.

Hirstionyssus carnifex (C. L. Koch) Oudemans, 1913

Umgebung Innsbruck, Talpa europaea: 2 m 62 f 1NII (XI). 
Nach Strandtmann et Wharton (1958) kommt die Art auf Clethrionomys lareolus, Apodemus flavicollis und anderen Kleinsäugern vor; in Mitteleuropa connte sie hauptsächlich auf Talpa europaea gefunden werden (MrCiAK et [OVORNIK 1966, MrCIAK et al. 1966).

Hirstionyssus tatricus Mrciak, 1958

Dergurgl, 2000-2100 m, Clethrionomys glareolus, Microtus nivalis : $20 \mathrm{~m} 87 \mathrm{f} 12 \mathrm{NII}$ (III, V, VIII, XI, XII). - Kühtai, 2250 m, Microtus nivalis : $1 \mathrm{f}$ (IX).

Es handelt sich hier um eine vorwiegend alpin auftretende Art, deren Hauptvirt Microtus nivalis ist. Sie wurde bisher nur aus der Hohen Tatra (CSR), den ;teiner Alpen (Jugoslawien), und dem Rila-Gebirge (Bulgarien) vorwiegend auf M. nivalis (MRCIAK 1959, 1964) und in Nordschweden gefunden (EDLER 1968).

Im jahreszeitlichen Auftreten ist ein Maximum aller Stadien im Winter u erkennen; allerdings stammt dieses Material von nur drei Wirten: Von je iner $C$. glareolus wurden am 14.XII $11 \mathrm{~m} 47 \mathrm{f} 8 \mathrm{NII}$ abgesammelt, am 17.III. - m 22 f 2 NII, am 17. Mai 3 m 10 f 2NII. Auf den übrigen Wirten (vier C. glareolus Ind vier $M$. nivalis) trat die Art nur in wenigen Exemplaren $(1-3)$ auf. In den Jntersuchungsgebieten wird $C$. glareolus gegenüber $M$. nivalis offensichtlich evorzugt: $5,2 \%$ der $C$. glareolus und 3\% der $M$. nivalis wurden als Wirte von 1. tatricus festgestellt, auf einer Rötelmaus konnte eine durchschnittliche Zahl on 0,8 Exemplaren H. tatricus gefunden werden, auf einer Schneemaus dagegen Iur 0,04 Exemplare! In einem typischen $M$. nivalis-Habitat, im Kühtai, wo Z. glareolus nicht mehr vorkommt, tauchte ein einziges Weibchen von H. tatricus uf einer von 81 untersuchten Schneemäusen auf.

Hirstionyssus eusoricis Bregetova, 1956

bergurgl, $2000 \mathrm{~m}$, Sorex alpinus : $3 \mathrm{f}$ (VI).

Diese nur aus wenigen Ländern Europas (Estland, Lettland, europäischer eil der USSR: Strandtmann et Wharton 1958; CSSR: Mrciak et Rosicky 956; Rumänien: FEIDER et al. 1965) bekannte Art wird vorwiegend auf Soriciden efunden und stellt vielleicht (wie $H$. soricis TURK) einen typischen Parasiten ieser Kleinsäuger dar.

Hirstionyssus musculi (Johnston, 1849)

Jmgebung Innsbruck, bis 1000 m, Apodemus flavicollis, Apodemus sp.: $11 \mathrm{f}$ (IV-VI, VIII). 
Ein typischer Parasit auf Vertretern der Gattung Apodemus, auf Microtine tritt die Art selten auf (MrCiaK et Brander 1965); in Gebieten, in denen Apodemus Arten fehlen, konnte $H$. musculi auf $M$. agrestis und $C$. glareolus gefunden werde (EDLER 1968).

\section{Hirstionyssus sp.}

Imst, Myotis oxygnathus : $1 \mathrm{~m}(\mathrm{X})$.

Myacarus arvicolae (Dujardin, 1849)

Umgebung Innsbruck, 1000 m, Clethrionomys glareolus: 15 Hypopus (III).

Verschiedene Kleinsäuger-Arten dienen als Wirt dieser Art, die auf de einzelnen Wirten oft in grösserer Zahl auftritt (MrClaK et BRANDER 1965 SAMSINAK 1957).

Ichoronyssus flavus (Kolenati, 1856)

Innsbruck, Nyctalus noctula: $4 \mathrm{f}$ 3NII (VIII).

I. flavus wurde von RAdovsKy (1967) unter die Synonyme von Macronyssu flavus (KolENATI) eingereiht; diese Art parasitiert vorwiegend auf $N$. noctulc und ist von diesem Wirt aus zahlreichen Ländern Europas bekannt geworder

Spinolaelaps jacksoni Radford, 1940

Imst, Myotis oxygnathus : $2 \mathrm{f}(\mathrm{X})$.

RADOVSKY (1967) betrachtet S. jacksoni als Synonym von Ichoronyssu scutatus (KolENATI, 1856) und führt eruopäische Fundorte aus Frankreich, de Tschechoslowakei und Russland an, als Wirte werden vorwiegend Myotis-Arte befallen. M. oxygnathus konnte als Wirt dieser Art im Libanon gefunden werder

Steatonyssus spinosus Willmann, 1936

Innsbruck, Nyctalus noctula: $1 \mathrm{f}$ (VIII). - Imst, Myotis oxygnathus : $2 \mathrm{f} 4 \mathrm{P}(\mathrm{X})$.

St. spinosus weist eine weite Verbreitung in der Palaearktis auf und konnt auf zahlreichen Fledermaus-Arten gefunden werden. In Europa ist sie allerding bisher nur aus Deutschland und der Tschechoslowakei bekannt gewese (RADOVSKY op. cit). 
Spinturnix acuminatus (C. L. Koch, 1836)

Innsbruck, Nyctalus noctula: $3 \mathrm{~m} 5 \mathrm{f}$ (VIII).

Nach Strandtmann et Wharton (1958) über ganz Europa verbreitet, als Hauptwirt wird der Abendsegler N. noctula angegeben.

Spinturnix myoti (Kolenati, 1858)

Imst, Myotis oxygnathus : $5 \mathrm{~m} 4 \mathrm{f} 5 \mathrm{~N}$ (X).

Ausser Fledermäusen der Gattung Myotis befällt diese Art auch zahlreiche andere Fledermausarten, aus Ländern Europas, und des Fernen Ostens, aus den USA und aus Nordafrika wurden Funde gemeldet (MrCiAK et NyHOLM 1967).

\section{Spinturnix sp.}

Schloss Tratzberg bei Schwaz, Myotis oxygnathus : $3 \mathrm{f}$ (IX).

\section{AUFTRETEN DER ARTEN IN DEN EINZELNEN MONATEN}

In dieser Aufstellung sind die (wenigen) Milben der Chiroptera nicht berücksichtigt, da diese Wirte nur zufällig und sporadisch abgesucht worden ware. Die Arten werden in alphabetischer Reihenfolge angeführt, in Klammern werden die Stückzahlen der einzelnen angetroffenen Stadien erwähnt.

JANUAR: C. mucronatus (2NII), E. stabularis $(1 \mathrm{~m})$, E. emarginatus (1NII), H. hirsutosimilis $(1 \mathrm{f}), H$. nidi $(5 \mathrm{f})$, L. agilis $(3 \mathrm{~m} 60 \mathrm{f} 2 \mathrm{NII})$, L. clethrionomydis $(1 \mathrm{f})$, P. necrophori (2NII) 8 Arten, 83 Exemplare.

FeBRUAR: E. lunulatus $(1 \mathrm{~m})$, E. remberti $(1 \mathrm{NII}), H$. hirsutosimilis $(8 \mathrm{f}), H$. nidi $(22 \mathrm{f}$ 3NII), L. agilis (2 m $27 \mathrm{f} \mathrm{2NII),} \mathrm{L.} \mathrm{clethrionomydis} \mathrm{(4} \mathrm{m} 8 \mathrm{f})$, L. hilaris $(1 \mathrm{~m} 1 \mathrm{f})$ 7 Arten, 80 Exemplare.

März: E. remberti (2NII), E. stabularis (1 f), H. nidi (1 m $3 \mathrm{f}), H$. tatricus $(4 \mathrm{~m} 22 \mathrm{f} 2 \mathrm{NII})$, H. arvalis (13 m $25 \mathrm{f} 3 \mathrm{NII})$, L. agilis $(1 \mathrm{f})$, L. clethrionomydis $(25 \mathrm{~m} 177 \mathrm{f} 15 \mathrm{NII})$, L. hilaris (3 m 67 f), M. muscaedomesticae (1 f), M. arvicolae (15 Hyp.), Parasitus sp. (16NII).

11 Arten, 396 Exemplare.

APRIL: C. minor (1NII), C. mucronatus (3NII), E. remberti (6NII), E. stabularis $(3 \mathrm{~m})$, E. emarginatus (3NII), H. hirsutosimilis (1 f), H. hirsutus (2NII), H. nidi $(5 \mathrm{~m} 24 \mathrm{f}$ $6 \mathrm{NII}), H$. isabellinus (1NII), H. musculi $(1 \mathrm{f}), H$. arvalis $(11 \mathrm{~m} 37 \mathrm{f} 7 \mathrm{NII} 2 \mathrm{NI})$, 
L. agilis (2 m $35 \mathrm{f} 4 \mathrm{NI})$, L. clethrionomydis (4 m $109 \mathrm{f} 6 \mathrm{NII})$, L. hilaris (11 m 43 2NII 1NI), P. spinosus (1 f).

15. Arten, 331 Exemplare.

MAI: C. minor (5NII), C. mucronatus (3NII), E. remberti (11NII), E. stabularis (5 $1 \mathrm{NII})$, E. emarginatus (3NII), H. hirsutus (2NII), H. horridus $(1 \mathrm{f} 1 \mathrm{NII}), H$. nid (8 m $20 \mathrm{f} 2 \mathrm{NII}), H$. musculi (3 f), H. tatricus (4 m $14 \mathrm{f} 2 \mathrm{NII}), H$. arvalis $(8 \mathrm{~m} 10 \mathrm{f}), L$ agilis (11 m $47 \mathrm{f} 7 \mathrm{NII} 2 \mathrm{NI})$, L. clethrionomydis (1 m $89 \mathrm{f} 2 \mathrm{NII} 1 \mathrm{NI})$, L. hilaris $(2 \mathrm{n}$ $25 \mathrm{f} 1 \mathrm{NII})$, M. montanus (1 f), M. ingricus (1 f), P. pygmaeus $(2 \mathrm{f})$.

17 Arten, 295 Exemplare.

JUNI: A. sardous (3 f), C. minor (5NII), C. mucronatus (1NII), E. lunulatus (1 m),

E. remberti (14NII), Eugamasus sp. (1NII), E. stabularis (5 f), E. emarginatus (4NII) E. ostrinus (1 f), H. hirsutosimilis (2 f), H. hirsutus (3NII), H. horridus (1 m 8NII) $H$. nidi $(2 \mathrm{~m} 13 \mathrm{f} 1 \mathrm{NII}), H$. eusoricis $(3 \mathrm{f}), H$. isabellinus $(15 \mathrm{f}), H$. musculi $(6 \mathrm{f})$ H. arvalis $(1 \mathrm{~m} 5 \mathrm{f} 1 \mathrm{NII}), H$. heselhausi $(1 \mathrm{f}), L$. agilis $(1 \mathrm{~m} 12 \mathrm{f} 3 \mathrm{NII} 2 \mathrm{NI})$, L. cle thrionomydis (8f), L. hilaris (29f), Ologamasus sp. (1 f), Parasitus sp. (7NII), P. necro phori (2NII), $P$. claparedei (4 f), $P$. forcipatus (1 f), $P$. spinosus $(1 \mathrm{f}), V$. nemorensi. (2 f).

28 Arten, 170 Exemplare.

JULI: C. minor (2NII), E. lunulatus $(1 \mathrm{~m} 2 \mathrm{f})$, E. remberti (3NII), E. stabularis $(13 \mathrm{f})$ E. emarginatus (1NII), H. hirsutus $(1 \mathrm{~m}), H$. horridus (2NII), H. nidi $(1 \mathrm{~m} 33 \mathrm{f} 4 \mathrm{NII})$ $H$. isabellinus $(3 \mathrm{f}), H$. arvalis $(3 \mathrm{~m} 11 \mathrm{f} 1 \mathrm{NII}), H$. heselhausi $(1 \mathrm{f})$, L. agilis $(8 \mathrm{n}$ $22 \mathrm{f})$, L. clethrionomydis (4 $\mathrm{m} 9 \mathrm{f})$, L. hilaris (18 f 1NII), M. matrius (3 f), Parasitus $\mathrm{sp}$ (5NII).

16 Arten, 152 Exemplare.

AUGUST: C. minor (1NII), C. mucronatus (2NII), E. stabularis (6 f), E. emarginatu. (1NII), H. horridus (2NII), H. nidi $(12 \mathrm{f} 1 \mathrm{NII}), H$. isabellinus $(2 \mathrm{~m} 16 \mathrm{f}), H$. muscul $(1 \mathrm{f})$, H. tatricus $(1 \mathrm{~m} 1 \mathrm{f})$, L. agilis $(14 \mathrm{f})$, L. clethrionomydis $(2 \mathrm{f})$, L. hilaris $(5 \mathrm{n}$ $44 \mathrm{f} 5 \mathrm{NII}$ 2NI), L. muris (1 f), M. montanus (1 f), Macrocheles sp. (1 f), M. ingricu. (1 f), P. furcifer (1 f), Parasitus sp. (3NII), Pergamasus sp. (1 f), P. necrophori (1NII) 20 Arten, 128 Exemplare.

SEPTEMBER: C. mucronatus (11NII), E. lunulatus (1 f), E. remberti (1NII), E. stabulari. (3 m $6 \mathrm{f})$, E. emarginatus (4NII), H. horridus (1 m $1 \mathrm{NII}), H$. nidiformis $(2 \mathrm{f}), H$. nid $(1 \mathrm{~m} 2 \mathrm{f} 2 \mathrm{NII}), H$. isabellinus $(3 \mathrm{f}), H$. tatricus $(1 \mathrm{f}), H$. arvalis $(3 \mathrm{~m} 5 \mathrm{f} 1 \mathrm{NI})$, L. agili. ( $2 \mathrm{~m} 40 \mathrm{f})$, L. clethrionomydis (1 f), L. hilaris (1 m $56 \mathrm{f} 1 \mathrm{NII})$, Parasitus sp. (7NII) 15 Arten, 156 Exemplare.

OKTOBER: E. stabularis (9 f), H. nidi (4 f), L. agilis (5 f), Parasitus sp. (1NII). 4 Arten, 19 Exemplare.

NOVEMBER: C. minor (1NII), C. mucronatus (2NII), E. stabularis (2 m $4 \mathrm{f})$, E. emar ginatus (3NII), $H$. hirsutosimilis (13 f), $H$. hirsutus (1NII), H. nidi $(9 \mathrm{~m} 73 \mathrm{f} 1 \mathrm{NI})$ H. carnifex $(2 \mathrm{~m} 62 \mathrm{f} 1 \mathrm{NII}), H$. tatricus $(3 \mathrm{f})$, L. agilis $(2 \mathrm{~m} 16 \mathrm{f} 1 \mathrm{NII})$, L. clethrio nomydis (1 f), L. hilaris (1 $\mathrm{m} 13 \mathrm{f}), M$. rossicus $(5 \mathrm{f} 1 \mathrm{NII})$.

13 Arten, 217 Exemplare.

DEZEMBER: E. remberti (4NII), E. emarginatus (1NII), H. hirsutosimilis (1 f), H. nid (6 m $10 \mathrm{f} 1 \mathrm{NII})$, H. tatricus $(11 \mathrm{~m} 47 \mathrm{f} 8 \mathrm{NII})$, L. clethrionomydis $(28 \mathrm{~m} 68 \mathrm{f} 13 \mathrm{NII})$ 6 Arten, 198 Exemplare. 
In einem Vergleich der einzelnen Monatsfänge muss berücksichtigt werden, lass einige Arten nur in einem einzigen Fangjahr auftauchten, z.B. H. musculi m Jahre 1967; H. hirsutus konnte nicht im Jahr 1968 gefunden werden; Hypoaspis heselhausi fand ich wieder nur 1969. Diese Festellung trifft natürlich besonders auf ene Arten zu, die nur einmal auf einem Wirt gefunden werden konnten (16 Arten, die Fledermaus-Milben noch ausgenommen). Trotz dieser gewissen Uneinheitlichkeit der Monatsaufsammlungen scheint die Tatsache interessant, dass die in sinem Monat auf den Wirten angetroffene absolute wie relative Zahl von Milbenndividuen gegen den Sommer hin trotz höherer Zahl von untersuchten Wirten absinkt, die absolute Artenzahl jedoch (erwartungsgemäss) zunimmt. Im März wurden 37 Wirte untersucht und auf einem Wirt durchschnittlich 10,7 Milben gefunden; die Artenzahl pro Wirt betrug 0,3. Im Juni konnten auf einem Kleinsäuger 3,6 Milben und 0,5 Arten angetrof̧fen werden. Im August konnten auf einem Wirt nur 1,7 Milben, im November dagegen bereits wieder 3,8 Milben gefunden werden. Nach diesen Ergebnissen zu schliessen, wäre die günstigste Zeit, in der man sich den besten Überblick über die vorkommenden Arten der Milben verschaffen kann, der Spätfrühling und Frühsommer (Juni, Juli, August).

Die höchste Stückzahl auf einem Wirtindividuum konnte auf einer Gelbhalsmaus Apodemus flavicollis mit 94 Exemplaren angetroffen werden ( 7 Arten: E. emarginatus, C. mucronatus, E. stabularis, L. agilis, M. rossicus, H. nidi, $H$. hirsutosimilis; davon gehörten 60 Exemplare zu $H$. nidi). Sehr häufig konnten mehrere Arten auf einem Kleinsäugerexemplar gefunden werden, meist waren es 3-4 Arten ( $37 \mathrm{mal}$ bzw. $15 \mathrm{mal}$ ); auf sechs Wirtsindividuen wurden 5 Arten entdeckt, sechs Arten tauchten auf insgesamt fünf Kleinsäugern auf, zweimal wurden je sieben Milbenarten von einem Wirtsexemplar abgesammelt; von Pitymys subterraneus: E. emarginatus, C. minor, A. sardous, E. ostrinus, $H$. horridus, $H$. isabellinus, $H$. nidi : einnal 8 von Apodemus flavicollis : C. mucronatus, E. emarginatus, E. stabularis, H. hirsutosimilis, H. hirsutus, H. horridus, H. nidi und L. agilis.

Öfters kamen auf den Wirten die gleichen Milben-Artbündel vor. So konnten auf 18 der 27 als Milbenträger nachgewiesenen Microtus agrestis, H. arvalis und L. hilaris gemeinsam gefunden werden (beide Arten kamen noch auf einer Microtus nivalis und einer Microtus arvalis vor); auf acht $M$. agrestis traten nur diese zwei Arten auf. Nach der Häufigkeit des gemeinsamen Auftretens zu schliessen, dürften die beiden Arten in den Nestern der M. agrestis die günstigsten Vermehrungsbedingungen vorfinden. Laelaps hilaris konnte noch auf sechs Wirten in Verbindung mit $H$. isabellinus, dreimal zusammen mit $E$. stabularis, $H$. nidi und $H$. arvalis gefunden werden. Auf 13 Kleinsäugern lebten $L$. hilaris und $H$. nidi gemeinsam, das Artenbündel L. hilaris $-H$. nidi $-H$. arvalis konnte achtmal auf $M$. agrestis gefunden werden. Laelaps clethrionomydis parasitierte auf 33 Clethrionomys glareolus, auf 21 Wirten dieser Art stellte sie die einzige 
Milbenart dar; sechsmal tauchten L. clethrionomydis und H. tatricus gemeinsan auf, die Kombination $L$. clethrionomydis $-H$. tatricus $-H$. nidi wurde au drei Wirtstieren gefunden. Laelaps agilis war auf Apodemus am häufigsten begleite von $E$. stabularis u. E. remberti (sechsmal), von $E$. remberti $-H$. nidi (5), vor $H$. nidi (8); gemeinsam mit $H$. hirsutosimilis wurde sie auf sieben Wirten gefunden mit $H$. horridus auf sechs und mit $H$. hirsutus auf vier Apodemus-Wirten. Die Ar Eugamasus remberti konnte auf acht Wirtsindividuen gemeinsam mit E. stabulari. entdeckt werden. Die meisten übrigen Kombinationen tauchten nur auf ein ode zwei Wirten auf.

\section{VERTIKALVERBREITUNG}

Über die Verbreitung in den einzelnen Höhenstufen können selbstverständ lich keine endgültigen Angaben gegeben werden, da einerseits fast keine Fäng zwischen $1000 \mathrm{~m}$ und $2000 \mathrm{~m}$ vorliegen, andererseits aber die höchsten vereinzelter Fänge nur aus 2600 m stammen. Schlüssige Aussagen über die Höhenverbreitun£ parasitischer Milben könnten überhaupt nur anhand zahlreicher Nestuntersuch ungen in allen Höhenstufen gemacht werden, da die Methode des Absuchens de1 Wirte zu viele Fehler birgt; Nestuntersuchungen liegen aber nicht vor.

Sowohl in den Tallagen bis $1000 \mathrm{~m}$ wie auch in der subalpinen (und alpinen) Stufe konnten 11 Gattungen mit 14 (?) Arten auf den Wirten angetroffen werden Cyrtolaelaps minor, C. mucronatus, Eugamasus remberti, Eulaelaps stabularis Euryparasitus emarginatus, Hyperlaelaps arvalis, Haemogamasus horridus, Hir. stionyssus isabellinus. Laelaps clethrionomydis, L. hilaris, Macrocheles montanus Parasitus sp, und Pygmephorus spinosus.

Nur in tiefer liegenden Untersuchungsgebieten (vorwiegend Umgebung Innsbruck bis $1000 \mathrm{~m}$ ) traten 14 Milbengattungen mit 19 (?) Arten auf: Androlaelaps sardous, Eugamasus sp., E. lunulatus, Eviphis ostrinus, Haemogamasus hirsutosimilis, H. hirsutus, Hirstionyssus carnifex, H. musculi, Hypoaspis heselhausi. Laelaps agilis, L. muris, Macrocheles sp., M. muscaedomesticae, Myacarus arvicolae. Myonyssus rossicus, Ologamasus sp., Pachylaelaps furcifer, Poecilochirus necrophori und Veigaia nemorensis.

Manche dieser Arten, die in dem Material aus der subalpinen und alpinen Stufe nicht vorkommen, sind aus anderen Gebieten der Alpen in diesen Höhenstufen gefunden worden, so z.B. E. lunulatus in den Schweizer Alpen bis $2600 \mathrm{~m}$ (SCHWEIZER 1949, 1961) oder V. nemorensis in Tirol (SCHMÖLZER 1962, JAHN 1960). Hier treten also methodische Lücken in der Aufsammlung zu Tage, verstärkt noch durch die Tatsache, dass manche dieser Arten eher zufällig auf den Kleinsäugern gefunden werden können (M. muscaedomesticae oder P. necrophori z.B.). Einzig die Arten, die eine enge Bindung an die Gattung Apodemus (L. agilis, H. musculi 
und auch $H$. hirsutosimilis) aufweisen, könnten im Untersuchungsgebiet auf die unteren Höhenstufen beschränkt sein, da auch die Apodemus-Arten nur selten in die subalpine Stufe hinaufsteigen. MoHr (1938) fing vier Apodemus sylvaticus auf dem Patscherkofel bei Innsbruck (1900-1950 m), SCHÄFER (1935) berichtet von zwei A. sylvaticus aus Vent (Ötztal) aus $1893 \mathrm{~m}$. Hirstionyssus carnifex ist sicher auch in den Nestern der alpinen Maulwürfe zu erwarten, im Kühtai kommt T. europaea bis ca. $2000 \mathrm{~m}$ vor, WetTSTEIN (1925) stellte Maulwürfe sogar noch in $2400 \mathrm{~m}$ fest.

Ausschliesslich in der subalpinen und alpinen Stufe konnten 8 Gattungen mit 9 Arten gefunden werden: Haemogamasus nidiformis, Hirstionyssus eusoricis, H. tatricus, Macrocheles matrius ssp. n. ?, Myonyssus ingricus, Pergamasus sp., Poecilochirus necrophori, Proctolaelaps pygmaeus und Protomyobia claparedei. Von diesen neun Arten sind nach der bisherigen' Kenntnis der Verbreitung nur die zwei Arten Haemogamasus nidiformis und Hirstionyssus tatricus als ausgeprägte Gebirgsformen anzusprechen. Als eine dritte Art mit einem deutlichen Schwerpunkt in der subalpinen Stufe ist auch Laelaps clethrionomydis zu werten. Diese Art konnte in den Steiner Alpen (Jugoslawien) nur mehr vereinzelt festgestellt werden, was MRCIAK (1959) darauf zurückführt, dass diese Art „höchstwahrscheinlich in diesem Alpengebiet die Grenze ihres südwestlichen Areals in Europa erreicht".

Hirstionyssus tatricus wurde bisher nur in vier Ländern gefunden, (Tschechoslowakei, Jugoslawien, Bulgarien, und Schweden), in Mittel-und Osteuropa meist in grossen Höhen vergesellschaftet mit Microtus nivalis; in Schweden fehlt Microtus nivalis, als Wirte konnten Clethrionomys rufocanus, Microtus agrestis, Lemmus lemmus und Mustela minuta entdeckt werden (EDLER 1968). MrCIAK (1959) hatte bereits darauf hingewiesen, dass $H$. tatricus vermutlich mit der Schneemaus in den Gebirgsgebieten des Karpatenbogens und des Alpensystems verbreitet sei. Aufgrund des Vorkommens in Schwedisch Lappland (EDLER op. cit) kann man vermuten, dass $H$. tatricus eine Art mit arktoalpiner Verbreitung darstellt.

Gleichfalls mit Microtus nivalis vergesellschaftet scheint Haemogamasus nidiformis zu sein. Die Art ist aus Bulgarien, der Tschechoslowakei und der USSR (MRCIAK 1959) vorwiegend aus Gebirgsgebieten bekannt, EDLER (1968) fand sie auf verschiedenen Kleinsäugern in Höhen zwischen 400 und $700 \mathrm{~m}$. MrCIAK (op. cit.) zählt $H$. nidiformis zu den ausgesprochenen Gebirgsformen.

Die Schneemaus Microtus nivalis ist meines Wissens bisher als Wirt von 16 Milbenarten bekannt geworden: Eulaelaps stabularis, Euryparasitus emarginatus, Haemogamasus nidi, $H$. nidiformis, $H$. bregetovae, H. hirsutus, Hirstionyssus isabellinus, H. tatricus, Hyperlaelaps arvalis, Laelaps hilaris, L. muris, Listrophorus leuckarti Pagenstecher, L. pagenstecheri Haller, Otonyssus sp., Poecilochirus necrophori und Dermacarus hypudaei Koch (Kahmann et HalbGewachs 1962; 
Mohr 1938, 1954; Mrciak 1959; Mrciak et Rosicky 1956). Strandtmann e WHARTON (1958) geben von M. nivalis nur eine Art an: Laelaps hilaris !

Folgende 7 Gattungen bzw. Arten, die im Laufe der Untersuchungen ir Nordtirol auf Microtus nivalis gefunden werden konnten, dürften demnach neut Parasiten dieser Kleinsäuger-Art darstellen: Cyrtolaelaps mucronatus, C. minor Eugamasus remberti, Macrocheles montanus, Parasitus sp., Pergamasus sp. unc Proctolaelaps pygmaeus. Damit sind mindestens 23 Arten parasitischer Milben (exclusive Trombiculidae u. Ixodidae) auf der Schneemaus festgestellt.

\section{ZUSAMMENFASSUNG}

Zwischen Herbst 1966 und Sommer 1969 wurden in Nordtirol von 664 Kleinsäuger aus verschiedenen Höhenstufen $(600 \mathrm{~m}-2600 \mathrm{~m})$ ektoparasitische Milben (excl. Ixodoidea) abgesammelt. 46 Arten, z.T. neu für Tirol und für Österreich, werden berichtet, für zahlreiche Arten konnten ergänzende Angaben zu ihrer Verbreitung gegeben werden (z.B. für Laelaps clethrionomydis, Myonyssus rossicus, Haemogamasus nidiformis, Hirstionyssus tatricus und $H$. eusoricis). Veränderungen im Auftreten einzelner Arten mit steigender Meereshöhe und im Wechsel der Jahreszeiten werden aufgezeigt Laelaps clethrionomydis, Haemogamasus nidiformis und Hirstionyssus tatricus traten beinahe ausschliesslich in der subalpinen und alpinen Stufe auf. Die durchschnittliche Individuenzahl der Acari pro Wirt nahm gegen den Sommer ab, die Zahl der Arten auf einem Wirt stieg dagegen an. Wirt-Parasit-Beziehungen einiger Arten werden diskutiert.

\section{RÉSUMÉ}

De l'automne 1966 à l'été 1969 les ectoparasites (Acariens excl. Ixodoidea) de 664 micromammifères provenant du Tirol septentrional (altitudes de 600 m-2600 m), ont été examinés. 46 espèces, en grande partie nouvelles pour le Tirol et l'Autriche, sont traitées. De nouvelles indications sur leur répartition sont données (p.e. pour Laelaps clethrionomydis, Myonyssus rossicus, Haemogamasus nidiformis, Hirstionyssus tatricus, $H$. eusoricis). Des modifications dans la présence de différentes espèces en fonction de l'altitude et des saisons sont signalées: Laelaps clethrionomydis, Haemogamasus nidiformis et Hirstionyssus tatricus ont été trouvés exclusivement dans l'étage subalpin et alpin; le nombre moyen des individus d'acariens par hôte diminue vers l'été, alors que le nombre d'espèces par hôte augmente. Les relations hôte-parasite de quelques espèces sont discutées. 


\section{SUMMARY}

Between autumn 1966 and summer 1969 ectoparasitic Acari had been collected of small mammals of different altitudinal zones in the Tirol (Austria). 46 species, most of them new for the Tirol or Austria, are recorded, for several species additional information on their distribution can be given (e.g. for Laelaps clethrionomydis, Myonyssus rossicus, Haemogamasus nidiformis, Hirstionyssus tatricus and $H$. eusoricis). Laelaps clethrionomydis, Haemogamasus nidiformis and Hirstionyssus tatricus only were found in the subalpine and alpine zone. The average number of mites per host decreased during summer, the average number of mite species on one host increased. Host-parasite-relations of some species are discussed.

\section{LITERATUR}

DANIEL, M. 1969. Nests of small terricolous mammals as the environment of nidicolous ectoparasites. Folia parasit. 16: 137-142.

EDLER, A. 1968. Ectoparasitic mites (Acarina: Parasitiformes) from small mammals from the Tärnasjö and Ammarnäs areas in Swedish Lapland. Entom. Ts. 89: $269-277$.

- 1969. Ectoparasitic mites (Acarina) from small mammals in Central Sweden. Entom. Ts. 90: 272-284.

Evans, G. O. 1957. An introduction to the British Mesostigmata (Acarina) with keys to families and genera. Linn. Soc. J. Zool. 93, Nr. 291: 203-259.

Feider, Z., L. Solomon und M. Hamar. 1965. Contributie la studiul parasitofaunei acarienilor de pe mamiferele mici din Republica Socialista Romania. Comun. Univ. "Al.I.Cuza“ Jassy: 297-309.

Franz, H. 1954. Die Nordost-Alpen im Spiegel ihrer Landtierwelt, Bd. I. Innsbruck, $664 \mathrm{~S}$.

JAHN, E. 1960. Ergebnisse von Bodentieruntersuchungen an der Wald- und Baumgrenze in Obergurgl. Cbl. ges .Forstwesen 77: 26-21.

- 1967. Ergebnisse bodenfaunistischer Untersuchungen an verschiedenen Lärchenstandorten Tirols. Ber. nat.-med. Ver. Innsbruck 55: 59-79.

Kahmann, H., J. Halbgewachs, 1962. Natürliche Feinde und Parasiten der Schneemaus Microtus nivalis (Martins), 1842. Z. Säugetierk. 27: 117-120.

KARG, W. 1961. Ökologische Untersuchungen an edaphischen Gamasiden (Acarina, Parasitiformes). 1. Teil. Pedobiologia 1: 53-74.

KePKA, O. 1964. Die Trombiculinae (Acari, Trombiculidae) in Österreich. Z. Parasitenk. 23: $548-642$.

LehmanN, E. v. 1969. Über die Hautdrüsen der Schneemaus (Chionomys nivalis nivalis Martins, 1842). Bonn. zool. Beitr. 20: 373-377. 
MAHNERT, V. 1970. Über Ento- und Ektoparasiten von Kleinsäugern der mittleren Ostalpen (Nordtirol). Diss. Phil. Fak. Univ. Innsbruck, 139 S.

- (im Druck): Parasitologische Untersuchungen an alpinen Kleinsäugern: Ixodoidea (Acari). Mitt. Schweiz. ent. Ges.

MoHr, E. 1938. Die Schneemaus in der Lebensgemeinschaft des Hochgebirges. Z. Naturwiss. 92: $67-85$.

- 1954. Die freilebenden Nagetiere Deutschlands und der Nachbarländer. Jena, viii $+212 \mathrm{~S}$.

MrciaK, M. 1959. Ein Beitrag zur Kenntnis der Milben (Parasitiformes) vom Kleinsäugern aus dem Gebiet Bulgariens. Prace brnenske zakladny CSAV: 31: $365-376$.

- 1960. Coniribution à la connaissance des acariens de l'ordre Parasitiformes en Albanie. Cesk. parasit. 7: 133-137.

- 1964. Beschreibung eines Männchens von Hirstionyssus tatricus MRCIAK 1958 (Acar : Liponyssidae). Biologia 19: 59-62 (tschech., dtsch. Zus.)

- und T. Brander. 1965. Beitrag zur Kenntnis der Milben (Acarina) einiger Säugetiere in Finnland. Lounais-Hämeen Luonto 17: 3-13.

- M. Daniel und B. Rosicky. 1966. Parasites and nest inhabitants of small mammals in the western Carpathians. I. Mites of the superfamily Gamasoidea (Parasitiformes). Acta F. R. N. Univ. Comen., Zool. 13: 81-116.

- und E. S. NyноLм. 1967. Beitrag zur Kenntnis der Milben der Familien Spinturnicidae im Gebiet von Rautalampi (Finnland). Lounais-Hämeen Luonto 25: 12-13.

- und B. Rosicky. 1956. Beiträge zur Fauna der Ordnung Parasitiformes (REUTER) ZACHVATKIN aus dem Gebiet der CSR. Zool. listy 19 (N.F. 5): 143-148 (tschech., dtsch. Zus.)

- und D. Tovornik. 1966. A contribution to the fauna of mites (Acari) from the territory of Slovenia (Yugoslavia). Acta F.R.N. Univ. Comen., Zool. 13: $117-139$.

Radovsky, F. J. 1967. The Macronyssidae und Laelapidae (Acarina: Mesostigmata) parasitic on bats. Univ. California Publ. Entom. 46: viii +288 S.

SAMSINAK, K. 1957. Einige ökologische Bemerkungen über die mit Säugetieren lebenden Milben der Überfamilie Tyroglyphoidea. Acta Soc. zool. bohem. 21: 93-94 (tschech., dtsch. Zus.).

SCHÄFER, H. 1935. Beitrag zur Kenntnis der Kleinsäugerfauna Tirols. Z. Säugetierk. 10: $154-155$.

SChmölzer, K. 1962. Die Kleintierwelt der Nunatakker als Zeugen einer Eiszeitüberdauerung. Mitt. zool. Mus. Berlin 38: 171-400.

SchweIzer, J. 1949. Die Landmilben des Schweizer Nationalparkes. 1. Teil: Parasitiformes REUTER 1909. Erg. wiss. Unters. SNP, 2 (N.F.), 21: 99 S.

- 1961. Die Landmilben der Schweiz (Mittelland, Jura und Alpen). Parasitiformes Reuter. Denkschr. Schweiz. Naturforsch. Ges. 84: 207 S.

Strandtmann, R.W. und G.W. Wharton. 1958. A manual of mesostigmatid mites parasitic on vertebrates. Inst. Acarol. Contrib. 4: $330 \mathrm{~S}$.

Wettstein-Westersheimb, O. 1925. Beiträge zur Säugetierkunde Europas I. Arch. Naturgsch., Abt. A, 91: 139-163.

Willmann, C. 1951. Untersuchungen über die terrestrische Milbenfauna im pannonischen Klimagebiet Österreichs. SB. öst. Akad. Wiss. Wien, math. nat. Kl., Abt. I., 160: 91-176. 
Villmann, C. 1952. Parasitische Milben an Kleinsäugern. Z. Parasitenk. 15: 392-428 1953. Neue Milben aus den östlichen Alpen. SB. öst. Akad. Wiss. Wien. math. —nat. K1., Abt. I., 162: 449-519.

Anschrift des Verfassers: Dr. Volker MahNERT, Muséum d'Histoire naturelle, Départeıent des Vertébrés inférieurs, route de Malagnou, CH-1211 Genève 6. 


\section{$2 \mathrm{BHL}$ Biodiversity Heritage Library}

Mahnert, V. 1971. "Parasitologische Untersuchungen an alpinen Kleinsäugern: Parasitische Milben (Acari)." Revue suisse de zoologie 78, 909-935. https://doi.org/10.5962/bhl.part.97083.

View This Item Online: https://www.biodiversitylibrary.org/item/138401

DOI: https://doi.org/10.5962/bhl.part.97083

Permalink: https://www.biodiversitylibrary.org/partpdf/97083

\section{Holding Institution}

American Museum of Natural History Library

\section{Sponsored by}

BHL-SIL-FEDLINK

\section{Copyright \& Reuse}

Copyright Status: Public domain. The BHL considers that this work is no longer under copyright protection.

Rights Holder: Muséum d'histoire naturelle - Ville de Genève

This document was created from content at the Biodiversity Heritage Library, the world's largest open access digital library for biodiversity literature and archives. Visit BHL at https://www.biodiversitylibrary.org. 